\title{
Solvent Remodeling in Single-Chain Amphiphilic
}

\section{Heteropolymer Systems}

\author{
Shayna L. Hilburg ${ }^{\dagger}$, Alfredo Alexander-Katz, ${ }^{\dagger *}$
}

Department of Materials Science and Engineering, Massachusetts Institute of Technology,

Cambridge, Massachusetts 02139, USA

6 ABSTRACT

7 Through molecular dynamics simulations, we demonstrate how single-chain nanoparticles

8 (SCNPs) assembled via transient linkages in water can remodel in organic solvent. Methacrylate-

9 based random heteropolymers (RHPs) have shown promise in an assortment of applications that

10 harness their bio-inspired properties. While their molecular behavior has been broadly

11 characterized in water, many newer applications include the use of organic solvent rather than bio-

12 mimetic conditions in which the polymer assemblies, typically driven by the hydrophobic effect,

13 are less well understood. Here, we examine a specific RHP system which forms compact globular

14 morphologies in highly polar and non-polar environments while adopting extended conformations

15 in solvents of intermediate polarity. We also demonstrate the pivotal role of electrostatic

16 interactions between charge groups in low dielectric mediums. Finally, we compare high

17 temperature anneal cycles to room temperature equilibrations to illuminate activation barriers to

18 remodeling upon environmental changes. 
Single-chain nanoparticles (SCNPs) harness synthetic macromolecules as promising drug

21 delivery systems, nanomedicines, catalysts, and more. ${ }^{1}$ While many SCNPs rely on covalent or

22 other strongly associating linkages to assemble, a promising subset of materials use reversible,

23 dynamic associations in their assembly. ${ }^{2}$ One of the simplest and most cost effective SCNP designs

24 is the combination of moieties with varying interaction energies which use solvophobic effects as

25 a driving force to assembly. Methacrylate-based random heteropolymers (RHPs) are one such

26 system, which self-assembles into nano-scale particles containing single or few chains in water,

27 and offers an exciting avenue for protein stabilization and mimicry, nanofiltration, and other

28 promising applications..$^{3-7}$. Behavior of methacrylate-based designs in water have been studied

29 most in depth, as many of their applications occur in aqueous environments. ${ }^{8,9}$

We previously studied a specific RHP design-space through molecular dynamics (MD)

31 simulations and elucidated the resulting SCNP structure and dynamics. ${ }^{10}$ The work demonstrated

32 that while theories are helpful in understanding these polymer systems, the inclusion of specific

33 chemistries is necessary for a full picture of the molecular behavior. While the work has been

34 pivotal in gaining a mechanistic picture of how the RHPs behave in water, several potential

35 applications occur in, or require processing steps performed in, other solvents. The compactness

36 and mobility of chains in solvents with different affinities for the varied chemical substituents has

37 been leveraged to control structures of resulting membranes, gels, and other nanomaterials. ${ }^{7,11,12}$

38 Characterizing the relationship between polymer behavior and its solvent environment allows such

39 methods to be applied predictably for a variety of polymer-solvent systems.

40 While covalently cross-linked SCNPs would not be considerably altered by the presence

41 of organic solvent, we expect drastic changes in systems, including ours, driven by transient 
42 linkages reliant upon solvent interactions to drive assembly. Amphiphilic synthetic polymer

43 systems which form compact assemblies in water can adopt extended conformations in solvents

44 of intermediate polarity, within which both hydrophobic and hydrophilic components energetically

45 prefer the enthalpic contributions to solvation over the entropic restriction of compactification, or

46 reverse micelles in solvents which invert typical aqueous behavior, leading hydrophilic

47 components to invert together while hydrophobic moieties preferentially orient to an assembly's

48 surface. This behavior has been experimentally observed for amphiphilic homopolymers with

49 amphiphilic monomers, ${ }^{13,14}$ as well as for heteropolymers with both hydrophobic and hydrophilic

50 monomers, most well characterized for 2-component copolymer systems. ${ }^{15-19}$ The specific

51 intramolecular interactions dictate how each polymer will adapt, with some systems maintaining

52 monomer contacts in organic media compared to aqueous solvent due to strong intrachain

53 associations which persist in the new environment. ${ }^{19,20}$

54 Our particular polymer of interest has been used to stabilize enzymes for the creation of

55 sustainably degradable polyesters through solution-casting in toluene or dichloromethane. ${ }^{21}$ In the

56 current work, we leverage our previously demonstrated simulation methods and broaden them to

57 a variety of organic solvents, namely tetrahydrofuran (THF), dimethyl sulfoxide (DMSO), and

58 hexane. We use atomistic molecular dynamics (MD) simulations, having seen the importance of

59 incorporating this level of chemical resolution through our prior work, as well as within the

60 literature. While coarse-grained simulations have been useful for RHP systems, such as in

61 demonstrating the correlation between stronger monomer interactions and smaller, denser

62 nanoparticle formation in copolymers, ${ }^{22}$ the methods require a fixed polarity and lack the atomic

63 solvent structures, making inherently amphiphilic molecular substituents such as poly(ethylene

64 glycol) difficult to model as they interact. Including chemical detail is vital to capturing the 
65 configurational entropy for side chain reorientation when transferring solvents and maintaining

66 separate assessment of backbone and side chain segments, which depending on their affinities to

67 the solvent will greatly alter resulting morphologies. ${ }^{23,24}$ In addition to our atomistic simulations of

68 the four-component RHPs in water, atomistic resolution has been leveraged in simulating two-

69 component amphiphilic copolymers in both water and chloroform. ${ }^{25} \mathrm{We}$ use similar techniques on

70 our more complex system with more in-depth analysis and demonstrate the impact of added

71 compositional complexity in a broader variety of solvents. The results we present show substantial

72 differences in monomer accessibility and conformational flexibility which depend on solvent

73 polarity and structure, as well as RHP chemistry. These behaviors affect how the molecules would

74 interface during post-synthetic processing steps or at end application in non-aqueous solvents and,

75 therefore, greatly impact their use. Beyond applications in organic solvent, our results also offer

76 significant insight to potential interactions with other organic substances such as small molecules

77 or hydrophobic biomacromolecules.

Herein, we explore RHP behavior using unbiased atomistic molecular dynamic methods

79 with the Amber MD package, similar to our prior work..$^{10,26}$ Through a series of successive anneal

80 cycles to $500 \mathrm{~K}$ in implicit water, ten conformations each of two distinct polymer sequences were

81 created and then annealed to $650 \mathrm{~K}$ in explicit solvent: water, DMSO, THF, or hexane. The

82 resulting single chain conformations at $300 \mathrm{~K}$ from these simulations were used for analysis.

83 Equilibrated structures from explicit water were then transferred to DMSO, THF, or hexane for

84 further study at room temperature as they adjust to the new solvent environments. Parameterization

85 and simulation details are provided in the Supplementary Information.

86 The two random heteropolymer sequences investigated, Sequence 1 and Sequence 2 as shown

87 in Figure 1, are composed of the methacrylate-based building blocks studied previously. ${ }^{10}$ 
88 Sequence 1 contains all four monomer species - methyl methacrylate (MMA), oligo(ethylene 89 glycol)methacrylate (OEGMA), 2-ethylhexyl methacrylate (EHMA), and 3-sulfopropyl

90 methacrylate (SPMA) - used in the prior work, while Sequence 2 is similar but lacks the anionic

91 SPMA monomer.

\section{Sequence 1}

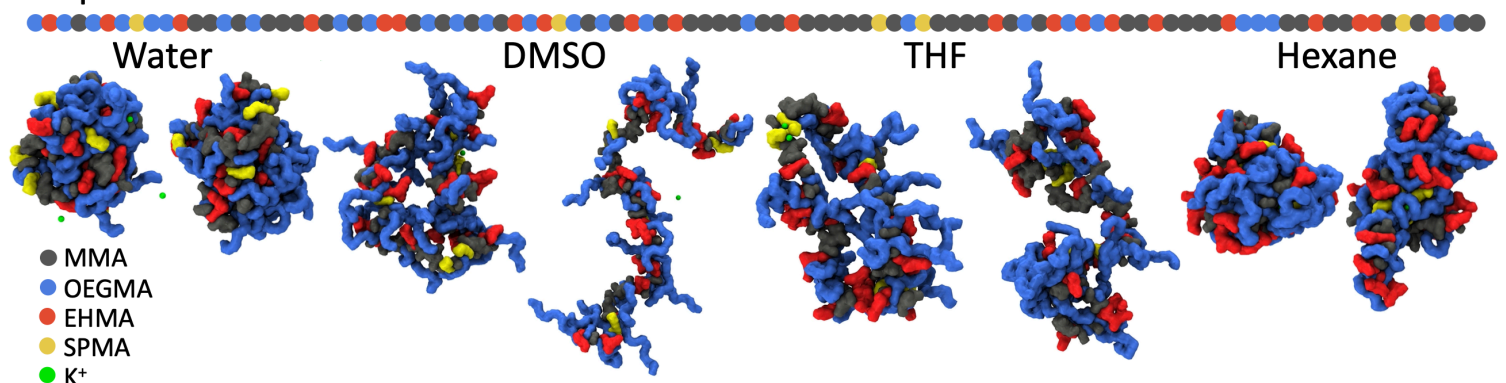

\section{Sequence 2}

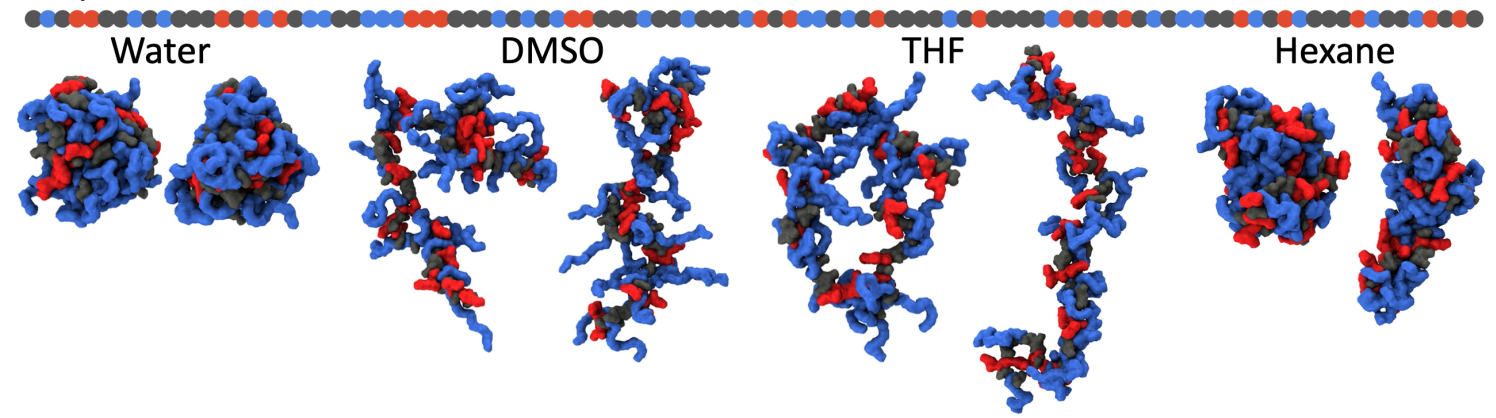

93 Figure 1. Sequences and renderings of the conformations with the minimum (left) and maximum

94 (right) mean radius of gyration for each system. Solvent is omitted for clarity.

Both sequences formed compact globular structures in water and hexane, with distinct

96 surface compositions in each solvent apparent by visual inspection in Figure 1. DMSO and THF

97 led to contrastingly extended conformations. Quantitatively, the mean radius of gyration $\left(\mathrm{R}_{\mathrm{g}}\right)$ and

98 solvent accessible surface area (SASA) for each equilibrated conformation is presented in Figure

99 2. The $\mathrm{R}_{\mathrm{g}}$, from which a minimum and maximum were selected for rendering in Figure 1 , has a

100 narrow distribution for compact structures in water and broader distributions for the organic

101 solvents of interest. SASA, calculated in each solvent using Amber's default radius size to allow 
102 cross-comparison, shows similar distributions within each dataset, indicating that the 103 configurations as adopted for the interaction energies between solvent and polymer have similar 104 amounts of contact despite entropically unique topologies indicated by unique $\mathrm{R}_{\mathrm{g}}$ values. Sequence 1051 and 2 have similar ranges for both properties in each examined environment, with the exception 106 of THF solvated structures which shows lower extension and solvation of sequence 1.

A.

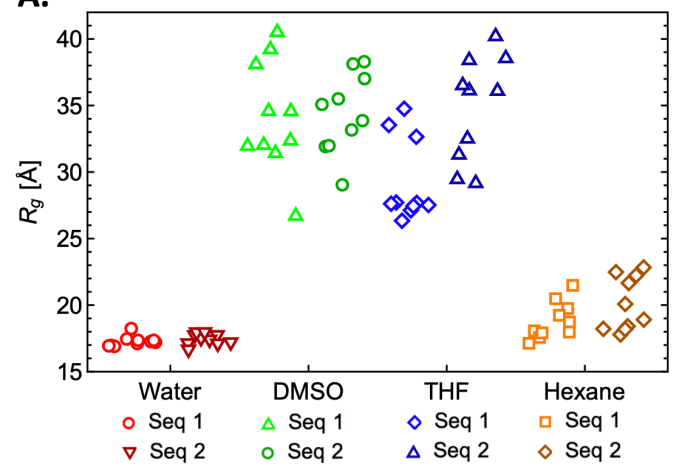

B.

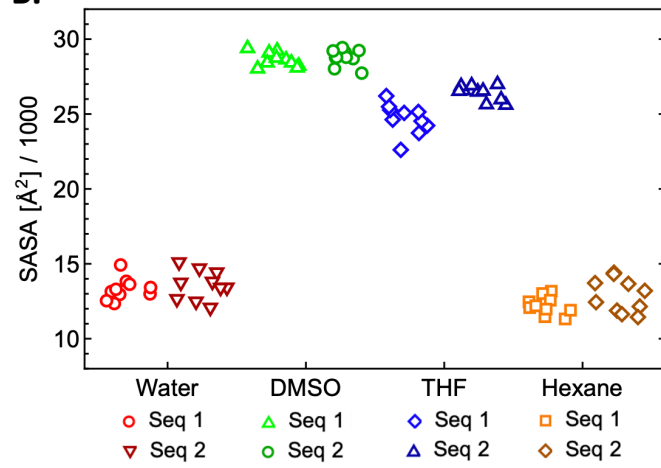

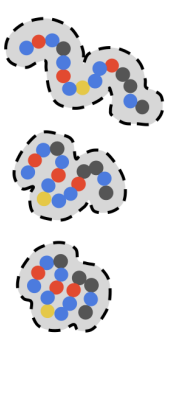

Figure 2. (A) Radius of gyration (Rg) and (B) total solvent accessible surface area (SASA) with

109 schematic representation (right) of how conformation and accessible surface area relate. Results

110 represent the mean value from equilibrated structures after annealing in solvent of interest for each

111 conformation.

Mobility of the polymer backbone and side-chains generally scales with chain extension,

113 albeit with several notable trends by monomer type (Supplementary Figures 1-2). While mobility

114 in THF is high and mobility in hexane is low, corresponding to the compactness, low backbone

115 mobility in SPMA monomer residues lead to lower values for Sequence 1 as compared to Sequence

1162 . Mobility of the side-chains themselves are impacted by moiety sizes, with OEGMA consistently

117 having high fluctuations in all solvents due to its long side-chain length, as well as being soluble

118 in water, THF, and DMSO. As EHMA and SPMA have similar side chain lengths, their relative

119 side-chain mobilities are indicative, instead, of freedom of motion in the polymer configuration. 
120 In water, SPMA fluctuates more than EHMA, while the trend is flipped in all three organic

121 solvents. In hexane, SPMA is practically immobilized and EHMA has significantly higher

122 fluctuations.

123 Polymer compactification and monomer mobilities all indicate that SPMA greatly impacts

124 chain behavior. An assessment of the radial distribution function (RDF) between sulfur atoms in

125 the SPMA's anionic sulfonate group, shown in Figure 3, shows that while some liquid-like features

126 can be seen between SPMA moieties in water, increasingly pronounced features and short

127 distances appear for the RHPs in DMSO, THF, and hexane. A similar analysis between sulfur and

128 potassium counterions (Supplementary Figure 3) indicates that salt bridging leads to these effects.

129 Intrachain associations have been observed in literature to restrict heteropolymer assembly through

130 salt bridging in water amongst anionic and cationic monomers and in organic solvent via strong

131 fluorocarbon associations. ${ }^{4,19}$ The extremely high correlations between sulfur positions in THF and

132 hexane in our conformations naturally explain the mobility and configurational differences

133 between sequence 1 and 2, locking sequence 1 into place due to electrostatic interactions in the

134 low dielectric mediums.

135

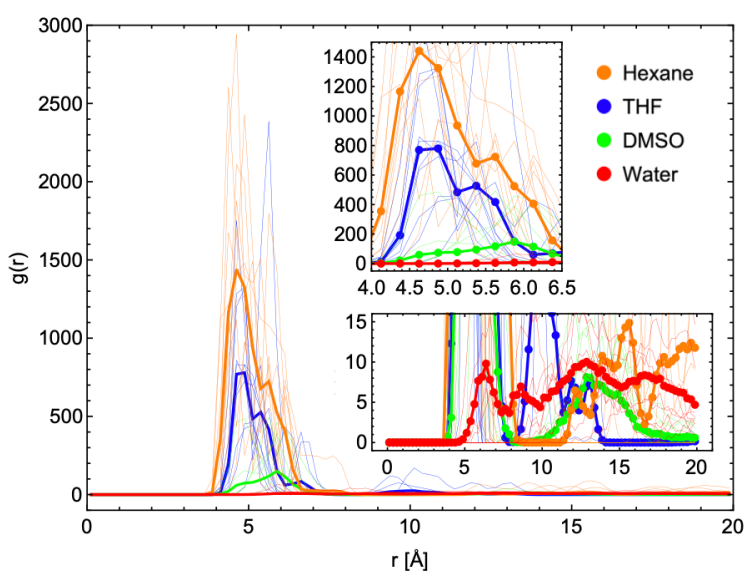


136 Figure 3. Radial distribution function of anionic moieties as measured between sulfur atoms within

137 the sulfonate groups of SPMA monomers. Bold lines show mean values over ten conformations

138 (each shown as thin lines) for the equilibrated sequence as annealed in each solvent of interest.

With equilibrated structures characterized, we turn to the transformation from water to

140 organic solvent. Data presented thus far annealed polymer chains in the explicit solvent of interest

141 before equilibrating for $60 \mathrm{~ns}$ at $300 \mathrm{~K}$, of which the final 40ns was used for the above analyses.

142 While some adjustment to the $\mathrm{R}_{\mathrm{g}}$ occurred at the beginning of equilibration following the anneal

143 (Supplementary Figure 4), the relative solvent accessibility (RSA) of each monomer type-the

144 partial SASA normalized by maximum monomer solvation (calculation details in Supplementary

145 Information) - stabilized quickly (Supplementary Figure 5). It should be noted that the resulting

146 trends between monomer species follow the aforementioned mobility data, with higher RSA for

147 monomers which showed greater fluctuations. Unexpectedly, however, monomer RSA is not

148 strictly correlated to overall chain extension and SASA, with SPMA residues showing significantly

149 lower accessibility in THF than in water despite adopting a conformation with nearly double the

$150 \mathrm{R}_{\mathrm{g}}$. If rather than being annealed in organic solvent, the polymers are instead annealed in explicit

151 water and then transferred to the solvent of interest for equilibration at room temperature, we find

152 that the same $60 \mathrm{~ns}$ is grossly insufficient for full remodeling. After $200 \mathrm{~ns}$, conformations in THF

153 and DMSO show increased $\mathrm{R}_{\mathrm{g}}$, though the majority remain far from corresponding equilibrium

154 values (Supplementary Figure 4). Figure 4 shows more rapid adaptation of RSA at room

155 temperature, with values trending towards equilibrium for THF and DMSO. Trends towards

156 equilibrium values indicate that kinetic barriers to rearrangement in THF and DMSO exist but can

157 be overcome through temperature - as in the annealed simulations - or time, as could be expected

158 if current trends continued in the room temperature simulations. Contrastingly, in hexane, RSA 
159 plateaus before reaching the equilibrium values. While behavior in hexane at room temperature is

160 observed to deviate from behavior in water, the assembly remains compact as $R_{\mathrm{g}}$ maintains values

161 below those of the configurations in DMSO and THF. The activation barrier to backbone

162 reconfiguration appears to trend with the driving force to compactification, and therefore the

163 changes observed in RSA seemingly approach a meta-stable kinetically-trapped state in which

164 side-chains have reoriented but the conformation is not entirely reconfigured. The property which

165 deviates from the water behavior most quickly at $300 \mathrm{~K}$ is the accessibility of SPMA in hexane.

166 This rapid change in RSA highlights the high energetic cost of sulfonate exposure to hexane and

167 the strong driving force for it to bury within the SCNP core, even if a full remodel is not

168 energetically favorable.
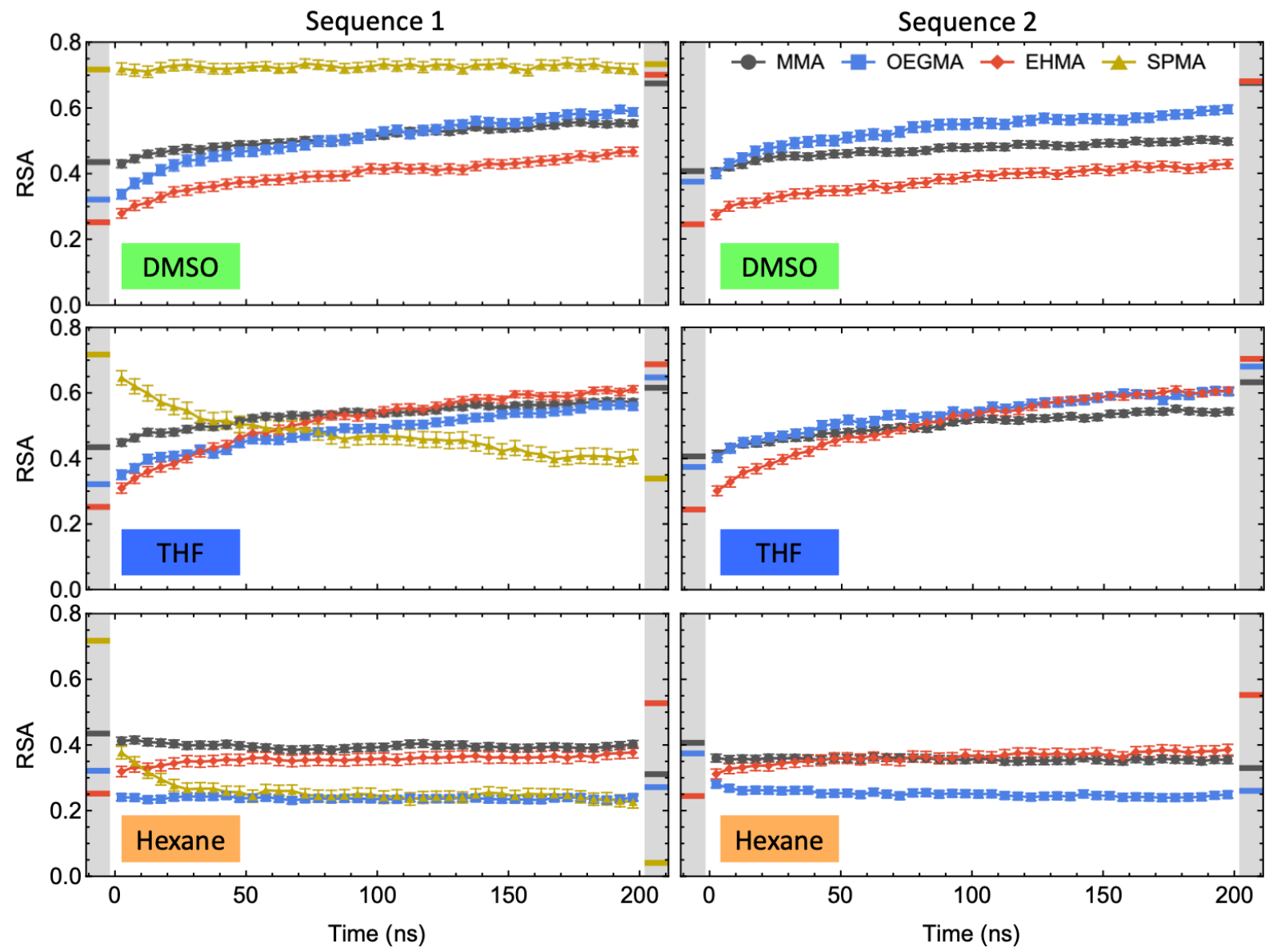
170 Figure 4. Change in relative solvent accessibility (RSA) of monomers over time after transfer to 171 solvent of interest at $300 \mathrm{~K}$. Each datapoint shows mean and standard error of the 5 ns average

172 RSA for each monomer of a given species within the sequence over all conformations. Shaded

173 regions on the left of each plot show mean RSA from conformations equilibrated in water while

174 shaded regions on the right of each plot show mean RSA from conformations equilibrated in the 175 solvent of interest (Supplementary Figure 5).

The results presented demonstrate that the covalently bound heterogenous monomers in 177 our RHP system will lead to compact globules with heterogeneous surfaces in both highly polar 178 (water) and highly nonpolar (hexane) solvents, though the driving forces differ greatly. The 179 hydrophobic effect, which drives assembly in water, leads to well solvated polar monomers with 180 more shielded hydrophobic moieties. In contrast, in hexane, the oleophobic polar and charged 181 groups avoid exposure and instead bury into the globule core, with hydrocarbon chemistries 182 prevailing at the assembly's surface. Therefore, the SCNP can exist in drastically different 183 configurations while still maintaining a compact geometry if the surface energy between the 184 solvent and monomers is high enough. Contrastingly, if entropic driving forces to polymer motion 185 outweigh the enthalpic costs to solvent exposure, the chain extends. In DMSO and THF, the 186 majority of monomers interact favorably with both solvents such that the RHP is generally open 187 and exposed. SPMA associations can limit extension and motion, but the small quantity of them 188 in the chain still affords enough freedom for other segments to maintain significant solvation. This 189 association nonetheless impacts accessibility to SPMA and its neighboring residues to solvent, 190 which has implications to post-synthetic modification strategies that could be employed. The 191 equilibrated structures illuminate the potential for interactions between RHPs or amongst RHPs 
192 and their environment in various settings, such as during processing in organic solvent to create 193 self-degrading polyesters or within the hydrophobic interior of cell membranes..$^{21,27}$

194 Beyond equilibrium behavior, our simulations also provide insight into how the polymer

195 chains get to those unique conformations and could behave upon transfer to distinct environments.

196 A change in solvent-monomer interaction energies can prompt either unfolding or remodeling,

197 with some reconfiguration possible without need for expansion to take place. Full reconfiguration

198 is best achieved using heat to overcome kinetic barriers on simulation timescales, though we also

199 demonstrated a near immediate adjustment to the new environment without thermal initiation.

200 In summary, the specific chemistry of the monomers selected for a heteropolymer as well

201 as their interactions with surrounding media prove to be of utmost importance to determining

202 polymer behavior. The structure which results from a given chemistry can lead to more traditional

203 SCNPs driven by hydrophobic interactions or other attractive and repulsive forces in certain

204 conditions while behaving as fully solvated, extended chains in others. The ratios of monomer

205 chemistries and the solvent polarities can be tailored to make specific moieties - and/or their

206 neighboring species - accessible or inaccessible. We have shown that reconfiguration can occur

207 significantly and rapidly with full remodeling possible at room temperature on timescales beyond

208 those typically accessible in simulation. This observed behavior has profound implications to how

209 the polymers may fold and assemble as they interface with proteins, surfaces, and other molecules

210 which impact intramolecular interactions.

\section{ASSOCIATED CONTENT}

212 Supporting Information. The Supporting Information is available on the XX website.

213 Simulation methods, backbone and side-chain mobility, sulfur-potassium RDF, and $\mathrm{R}_{\mathrm{g}}$ over time 214 (PDF) 


\section{AUTHOR INFORMATION}

\section{Corresponding Author}

$217 *$ aalexand@mit.edu

\section{Funding Sources}

219 This work was supported by the Defense Threat Reduction Agency contract HDTRA11910011.

\section{ACKNOWLEDGEMENTS}

221 We are thankful to Ting $\mathrm{Xu}$ and Marco Eres for insightful discussion in the preparation of this 222 work.

\section{REFERENCES}

224 (1) Alqarni, M. A. M.; Waldron, C.; Yilmaz, G.; Becer, C. R. Synthetic Routes to Single Chain 225 Polymer Nanoparticles (SCNPs): Current Status and Perspectives. Macromol. Rapid Commun. $226 \mathbf{2 0 2 1}, 154(15), 2100035$.

227 (2) Blazquez-martín, A.; Verde-sesto, E.; Moreno, A. J.; Arbe, A.; Colmenero, J. Advances in 228 the Multi-Orthogonal Folding of Single Polymer Chains into Single-Chain Nanoparticles. 2021, $229 \quad 1-17$.

230 (3) Gao, J.; Le, S.; Thayumanavan, S. Enzyme Catalysis in Non-native Environment with 231 Unnatural Selectivity Using Polymeric Nanoreactors. Angew. Chemie Int. Ed. 2021.

232 (4) Bhat, R.; Foster, L. L.; Rani, G.; Vemparala, S.; Kuroda, K. The function of peptide233 mimetic anionic groups and salt bridges in the antimicrobial activity and conformation of cationic 234 amphiphilic copolymers. RSC Adv. 2021, 11 (36), 22044-22056. 
(5) Lamm, R. J.; Pichon, T. J.; Huyan, F.; Wang, X.; Prossnitz, A. N.; Manner, K. T.; White,

236 N. J.; Pun, S. H. Optimizing the Polymer Chemistry and Synthesis Method of PolySTAT, an 237 Injectable Hemostat. ACS Biomater. Sci. Eng. 2020, 6 (12), 7011-7020.

238 (6) Panganiban, B.; Qiao, B.; Jiang, T.; DelRe, C.; Obadia, M. M.; Nguyen, T. D.; Smith, A. 239 A. A.; Hall, A.; Sit, I.; Crosby, M. G.; Dennis, P. B.; Drockenmuller, E.; Olvera de la Cruz, M.; $240 \mathrm{Xu}, \mathrm{T}$. Random heteropolymers preserve protein function in foreign environments. Science (80-. 241 ). 2018, $359(6381), 1239-1243$.

(7) Yu, W.-H.; Qiu, Z.-L.; Wang, J.-R.; Shen, Y.-J.; Han, J.; Fang, L.-F.; Zhu, B.-K. Novel nanofiltration membrane prepared by amphiphilic random copolymer nanoparticles packing for 244 high-efficiency biomolecules separation. Chem. Eng. J. 2022, 430, 132914.

(8) Verde-Sesto, E.; Arbe, A.; Moreno, A. J.; Cangialosi, D.; Alegría, A.; Colmenero, J.; 247 confinement. Mater. Horizons 2020, 7 (9), 2292-2313.

(9) Frisch, H.; Tuten, B. T.; Barner-Kowollik, C. Macromolecular Superstructures: A Future 249 Beyond Single Chain Nanoparticles. Isr. J. Chem. 2020, 60 (1-2), 86-99.

250 (10) Hilburg, S. L.; Ruan, Z.; Xu, T.; Alexander-Katz, A. Behavior of Protein-Inspired 251 Synthetic Random Heteropolymers. Macromolecules 2020, 53 (21), 9187-9199.

252 (11) Scheutz, G. M.; Elgoyhen, J.; Bentz, K. C.; Xia, Y.; Sun, H.; Zhao, J.; Savin, D. A.; 253 Sumerlin, B. S. Mediating covalent crosslinking of single-chain nanoparticles through 254 solvophobicity in organic solvents. Polym. Chem. 2021, 12 (31), 4462-4466. 
(12) Zhang, Y. Y.; Jia, X. M.; Shi, R.; Li, S. J.; Zhao, H.; Qian, H. J.; Lu, Z. Y. Synthesis of 256 Polymer Single-Chain Nanoparticle with High Compactness in Cosolvent Condition: A Computer 257 Simulation Study. Macromol. Rapid Commun. 2020, 41 (24), 1-7.

(13) Guazzelli, E.; Masotti, E.; Calosi, M.; Kriechbaum, M.; Uhlig, F.; Galli, G.; Martinelli, E. 259 Single-chain folding and self-assembling of amphiphilic polyethyleneglycol-modified fluorinated 260 styrene homopolymers in water solution. Polymer (Guildf). 2021, 231, 124107.

(14) Li, Q.; Constantinou, A. P.; Georgiou, T. K. A library of thermoresponsive PEG-based 262 methacrylate homopolymers: How do the molar mass and number of ethylene glycol groups affect 263 the cloud point? J. Polym. Sci. 2021, 59 (3), 230-239.

264 (15) Goswami, K. G.; Mete, S.; Chaudhury, S. S.; Sar, P.; Ksendzov, E.; Mukhopadhyay, C. 265 Das; Kostjuk, S. V.; De, P. Self-Assembly of Amphiphilic Copolymers with Sequence-Controlled 266 Alternating Hydrophilic-Hydrophobic Pendant Side Chains. ACS Appl. Polym. Mater. 2020, 2 (5), $267 \quad 2035-2045$.

(16) Sadeghi, I.; Asatekin, A. Spontaneous Self-Assembly and Micellization of Random 269 Copolymers in Organic Solvents. Macromol. Chem. Phys. 2017, 218 (20), 1-9.

270 (17) Uddin, M. A.; Yu, H.; Wang, L.; Naveed, K.-R.; Amin, B. U.; Mehmood, S.; Haq, F.; 271 Nazir, A.; Lin, T.; Chen, X.; Ni, Z. Multiple-stimuli-responsiveness and conformational inversion 272 of smart supramolecular nanoparticles assembled from spin labeled amphiphilic random 273 copolymers. J. Colloid Interface Sci. 2021, 585, 237-249. 
274 (18) Hattori, G.; Takenaka, M.; Sawamoto, M.; Terashima, T. Nanostructured Materials via the

275 Pendant Self-Assembly of Amphiphilic Crystalline Random Copolymers. J. Am. Chem. Soc. 2018, $276140(27), 8376-8379$.

277 (19) Koda, Y.; Terashima, T.; Sawamoto, M. Multimode Self-Folding Polymers via Reversible 278 and Thermoresponsive Self-Assembly of Amphiphilic/Fluorous Random Copolymers. 279 Macromolecules 2016, 49 (12), 4534-4543.

280 (20) Matsumoto, K.; Terashima, T.; Sugita, T.; Takenaka, M.; Sawamoto, M. Amphiphilic 281 Random Copolymers with Hydrophobic/Hydrogen-Bonding Urea Pendants: Self-Folding 282 Polymers in Aqueous and Organic Media. Macromolecules 2016, 49 (20), 7917-7927.

283 (21) DelRe, C.; Jiang, Y.; Kang, P.; Kwon, J.; Hall, A.; Jayapurna, I.; Ruan, Z.; Ma, L.; Zolkin, 284 K.; Li, T.; Scown, C. D.; Ritchie, R. O.; Russell, T. P.; Xu, T. Near-complete depolymerization of 285 polyesters with nano-dispersed enzymes. Nature 2021, 592 (7855), 558-563.

286 (22) Vao-soongnern, V. Molecular simulation of molecular and surface properties of random 287 copolymer nanoparticle. J. Mol. Liq. 2021, 342, 117556.

288 (23) Chawathe, M.; Patel, A.; Jonnalagadda, S.; Sidorenko, A. Design of hybrid molecular 289 brushes with reversible surface adaptability on exposure to specific solvents. Biointerphases $\mathbf{2 0 1 8 ,}$ $29013(4), 041006$.

291 (24) Buglakov, A. I.; Larin, D. E.; Vasilevskaya, V. V. Orientation- and cosolvent-induced self292 assembly of amphiphilic homopolymers in selective solvents. Polymer (Guildf). 2021, 232, 293124160. 
(25) Guazzelli, E.; Martinelli, E.; Galli, G.; Cupellini, L.; Jurinovich, S.; Mennucci, B. Single295 chain self-folding in an amphiphilic copolymer: An integrated experimental and computational 296 study. Polymer (Guildf). 2019, 161 (November 2018), 33-40.

297 (26) D.A. Case, I.Y. Ben-Shalom, S.R. Brozell, D.S. Cerutti, T.E. Cheatham, III, V.W.D. 298 Cruzeiro, T.A. Darden, R.E. Duke, D. Ghoreishi, G. Giambasu, T. Giese, M.K. Gilson, H. Gohlke, 299 A.W. Goetz, D. Greene, R Harris, N. Homeyer, Y. Huang, S. Izadi, A. Kovalenko, R. Krasny, T. 300 Kurtzman, T.S. Lee, S. LeGrand, P. Li, C. Lin, J. Liu, T. Luchko, R. Luo, V. Man, D.J. 301 Mermelstein, K.M. Merz, Y. Miao, G. Monard, C. Nguyen, H. Nguyen, A. Onufriev, F. Pan, R. 302 Qi, D.R. Roe, A. Roitberg, C. Sagui, S. Schott-Verdugo, J. Shen, C.L. Simmerling, J. Smith, J. 303 Swails, R.C. Walker, J. Wang, H. Wei, L. Wilson, R.M. Wolf, X. Wu, L. Xiao, Y. Xiong, D.M. 304 York and P.A. Kollman (2019), AMBER 2019, University of California, San Francisco.

305 (27) Jiang, T.; Hall, A.; Eres, M.; Hemmatian, Z.; Qiao, B.; Zhou, Y.; Ruan, Z.; Couse, A. D.; 306 Heller, W. T.; Huang, H.; de la Cruz, M. O.; Rolandi, M.; Xu, T. Single-chain heteropolymers 307 transport protons selectively and rapidly. Nature 2020, 577 (7789), 216-220. 